Research Paper

\title{
Retroperitoneal dedifferentiated liposarcoma: Analysis of 61 cases from a large institution
}

\author{
Peng Luo ${ }^{1,2^{*}}$, Weiluo Cai ${ }^{1,2^{*}}$, Lingge Yang ${ }^{1,2}$, Zhiqiang $\mathrm{Wu}^{1,2}$, Yong Chen ${ }^{1,2}$, Ruming Zhang ${ }^{1,2}$, Wangjun \\ Yan ${ }^{1,2}$, Yingqiang Shi ${ }^{1,2}$, Chunmeng Wang ${ }^{1,2}$ \\ 1. Department of Bone and Soft Tissue Sarcomas, Fudan University Shanghai Cancer Center, Shanghai, China \\ 2. Department of Oncology, Shanghai Medical College, Fudan University, Shanghai, China \\ *Peng Luo and Weiluo Cai contributed equally to this work. \\ $\triangle$ Corresponding author: Dr. Chunmeng Wang, MD, Department of Bone and Soft Tissue Sarcomas, Fudan University Shanghai Cancer Center; Department of \\ Oncology, Shanghai Medical College, Fudan University, Shanghai 200032, China. Fax: 86-21-64430130. E-mail: cmwang1975@163.com \\ (c) Ivyspring International Publisher. This is an open access article distributed under the terms of the Creative Commons Attribution (CC BY-NC) license \\ (https://creativecommons.org/licenses/by-nc/4.0/). See http://ivyspring.com/terms for full terms and conditions.
}

Received: 2018.02.25; Accepted: 2018.07.20; Published: 2018.10.05

\begin{abstract}
Background: To describe the clinical features of retroperitoneal dedifferentiated liposarcoma (RP DDLS) and further evaluate the prognostic factors.

Methods: The clinicopathological variables and treatment strategies of 61 RP DDLS patients who underwent surgical resections at a single institution between September 2005 and September 2016 were reviewed. Kaplan-Meier and Cox regression methods were conducted for survival analyses.

Results: The average patients' age was 52 years (range, 27-81), and there was almost no gender predilection ( 30 males vs. 31 females). 51 (83.6\%) patients got gross tumor resections (R0/R1 resection), and the median tumor size was $19 \mathrm{~cm}$ (range, 4.3-50 cm). 39(63.9\%) patients were with intermediate-grade sarcoma and $22(36.1 \%)$ were with high-grade sarcoma. The median intraoperative blood loss was $400 \mathrm{ml}$ (range, $50-2700 \mathrm{ml}) .19(31.1 \%)$ patients presented multifocal diseases. Tumors were removed intactly in $42(68.9 \%)$ patients. In order to obtain gross tumor resections, $33(54.1 \%)$ of the patients underwent excisions of at least one adjacent organ, of which kidney $(n=21,63.6 \%)$ was the most common one. $6(9.8 \%)$ patients developed distant metastases during follow-up. The overall 5-year progression-free survival (PFS) rate was $3.7 \%$, with the median PFS of 19 months. The 5-year overall survival (OS) rate was $43.5 \%$, with the median OS of 58 months. Updating to November 2017, 30 (49.2\%) patients remained alive. The median follow-up time was 49 months. Multivariate analysis using Cox proportional hazards model revealed that tumor grade, blood loss, resection extent, and tumor integrity were independently associated with OS $(p=0.032, p=0.018, p=0.020, p=0.005$, respectively). Tumor grade, tumor integrity and multifocality were significant predictors for PFS $(p=0.013, p=0.080, p=0.009, p=0.028$, respectively).

Conclusion: Intermediate-grade histology, intraoperative blood loss $<400 \mathrm{ml}$, complete tumor resection, and tumor integrity were independently associated with better OS. Intermediate-grade histology, tumor integrity and unifocal disease independently predicted favorable PFS.
\end{abstract}

Key words: retroperitoneal dedifferentiated liposarcoma, Asian, predictors

\section{Introduction}

Liposarcoma (LS) represents one of the most common soft tissue sarcomas (STS), accounting for nearly $20 \%$ of new diagnosis [1]. According to Evans Classification, LS can be classified into 5 subtypes: well-differentiated (WDLS), myxoid (MLS), dedifferentiated (DDLS), round-cell (RLS), and pleomorphic (PLS) [2]. Evans defined DDLS as WDLS with cellular non-lipogenic spindle cell or pleomorphic sarcoma, the spectrum of DDLPS was expanded to include WDLPS with low-grade 
dedifferentiation [3]. DDLS accounts for approximately $18 \%$ of all LS [4], while a recent study of 231 LS patients reporting $13.9 \%$ in Asian population [5].

Most commonly occurred in retroperitoneal (RP) [1], DDLS is a highly malignancy generally affecting adults in the 6th to 7 th decades of life [6]. The majority of $(90 \%)$ DDLPS arises de novo, whereas $10 \%$ developed from pure WDLS [7-9]. Based on different histologic subtype and different primary site, the natural history of LS can vary markedly from a locally recurrent tumor to a highly malignancy that bears a grim prognosis. However, hitherto much of the existing studies of LS are either site specific (ie, RP) [8, 9] or histology specific (ie, DDLS) [1, 7]. Only one RP DDLPS-specific literature analyzed the clinicopathologic features identify prognostic factors to facilitate clinical practice [9]. The scant of study focusing specifically on RP DDLPS of Asian population makes such research in urgent need.

Therefore, the aim of this study was to analyze the clinicopathologic features of RP DDLPS among Asian population and identify the prognostic factors using data collected from a single-center.

\section{Methods}

\section{Patient selection}

This study was approved by the Ethics Committee of Fudan University Shanghai Cancer Center and was performed in accordance with the approved guidelines. Written informed consent was obtained from all patients. The criteria for inclusion were as follows: (1) surgically resectable localized disease (without distant metastasis) at the time of diagnosis, patients with biopsy only were excluded (2) no severe surgical complications leading to death (One patient with severe intra-abdominal infection was excluded) (3) histologically confirmed diagnosis of DDLS (4) completeness of follow-up data and clinical information, including patient demographics, tumor size, tumor grade, blood loss, admission status, extent of resection, tumor integrity, concomitant organ resection, multifocality, adjuvant treatment records. Eventually, 61 patients with primary or recurrent RP DDLS who underwent surgical resections from September 2005 to September 2016 at Fudan University Shanghai Cancer Center were included in this study.

Multifocal disease was defined as having more than 1 distinct tumor nodules, as opposed to multiple DDLS contained within a solitary focus of WDLS [9]. Resection extent was classified into 2 groups, gross tumor resection (R0/R1) and palliative resection (R2 resection). Tumor burden was counted as the maximum diameter of tumor for unifocal disease or the sum of the maximum diameter of every single lesion for multifocal disease. Concomitant organ resections were only performed for directly involved adjacent organs or to facilitate expected gross tumor resections. Tumor grade was defined by the French Federation of Cancer Centers Sarcoma Group (FNCLCC) grading system [10]. The information of tumor burden, tumor grade, blood loss, concomitant organ resection, extent of resection, tumor integrity (intact vs piecemeal tumor resection) was determined by the operative report and/or pathology report. During the follow-up, patients who developed suspicious metastatic lesions were administered further imageological examinations such as contrast-enhanced CT, contrast-enhanced MR, ECT, or PET-CT. All available imagings of patients with metastatic disease were systematically reviewed in consensus by two experienced radiologists with expertise in cancer imagining. Pathological slides were independently confirmed by two experienced pathologists with expertise in soft tissue sarcomas.

\section{Statistical analysis and Follow-up data}

SPSS 21.0 was applied for statistical analysis. Median overall survival (OS) and progression-free survival (PFS) were determined using the Kaplan-Meier method. Blood loss and tumor size were dichotomized for Kaplan-Meier analysis [11]. Log-rank test was applied to comparing survival between groups, and Cox proportional hazards regression modeling was used to perform multivariate analysis. Only the factors identified statistically significant in the univariate analysis were then put in multivariate analysis. All tests were two-sided with a significance level set at $P<0.05$.

OS was counted as the interval from the date of surgery to the date of death (event) related to the disease (or complications). PFS was calculated from the date of surgery to the date of the first distant metastases, the first local recurrence, progression of residual lesion, or death without evidence of distant metastases, recurrence or progression. For patients alive and without records of disease relapse (local recurrence/progression or distant metastases), follow-up was censored at the time of last follow up. Follow-up data was collected by phone call or outpatient records. All 61 patients were continuously followed up to either November 2017, the time of final follow-up, or the date of death.

\section{Results}

\section{Patient characteristics}

In this study, the comprehensive data of $61 \mathrm{RP}$ DDLS patients underwent surgeries with curative 
intent at a Fudan university Shanghai cancer center between September 2005 and September 2016 were analyzed. Data including age, sex, tumor burden, blood loss, multifocality, admission status, extent of resection, tumor integrity, concomitant organ resection, adjuvant treatment, and postoperative metastases were listed in Table 1.

Table 1. Table 1 Patients' characteristics.

\begin{tabular}{|c|c|c|c|}
\hline Variable & $\begin{array}{l}\text { Total }(\mathrm{N}=61 \text {, } \\
\% \text { or range) }\end{array}$ & Primary(N=30) & $\begin{array}{l}\text { Recurrent }(\mathrm{N}= \\
\text { 31) }\end{array}$ \\
\hline \multicolumn{4}{|l|}{ Gender } \\
\hline Male & $30(49.2)$ & $13(43.3 \%)$ & 17 \\
\hline Female & $31(50.8)$ & $17(56.7 \%)$ & 14 \\
\hline Age (year, median) & $52(27-81)$ & $54(36-75)$ & $50(27-81)$ \\
\hline$<50$ & $24(39.3)$ & $10(33.3 \%)$ & 14 \\
\hline$\geq 50$ & $37(60.7)$ & $20(66.7 \%)$ & 17 \\
\hline $\begin{array}{l}\text { Tumor burden }(\mathrm{cm}, \\
\text { median) }\end{array}$ & $19(4.3-50)$ & $20(7-50)$ & $18(4.3-33)$ \\
\hline$<19 \mathrm{~cm}$ & $29(47.5)$ & $12(40 \%)$ & 17 \\
\hline$\geq 19 \mathrm{~cm}$ & $32(52.5)$ & $18(60 \%)$ & 14 \\
\hline \multicolumn{4}{|l|}{ Grade } \\
\hline Intermediate & $39(63.9 \%)$ & $17(56.7 \%)$ & $22(71 \%)$ \\
\hline High & $22(36.1 \%)$ & $13(43.3 \%)$ & $9(29 \%)$ \\
\hline Blood loss (ml, median) & $400(50-2700)$ & $400(50-2700)$ & $300(50-2500)$ \\
\hline$<400 \mathrm{ml}$ & $28(45.9)$ & $12(40 \%)$ & 16 \\
\hline$\geq 400 \mathrm{ml}$ & $33(54.1)$ & $18(60 \%)$ & 15 \\
\hline \multicolumn{4}{|l|}{ Admission status } \\
\hline Primary & $30(49.2)$ & - & - \\
\hline Recurrent & $31(50.8)$ & - & - \\
\hline \multicolumn{4}{|l|}{ Extent of resection } \\
\hline R0/R1 & $51(83.6)$ & $27(90 \%)$ & 24 \\
\hline $\mathrm{R} 2$ & $10(16.4)$ & $3(10 \%)$ & 7 \\
\hline \multicolumn{4}{|l|}{ Tumor integrity } \\
\hline Intact & $42(68.9)$ & $22(73.3 \%)$ & 20 \\
\hline Piecemeal & 19(31.1) & $8(26.7 \%)$ & 11 \\
\hline \multicolumn{4}{|l|}{ Organ resection } \\
\hline No & $28(45.9)$ & $15(50 \%)$ & 13 \\
\hline Yes & $33(54.1)$ & $15(50 \%)$ & 18 \\
\hline \multicolumn{4}{|l|}{ Multifocality } \\
\hline No & $42(68.9)$ & $24(80 \%)$ & 18 \\
\hline Yes & $19(31.1)$ & $6(20 \%)$ & 13 \\
\hline \multicolumn{4}{|l|}{ Adjuvant treatment } \\
\hline No & $44(72.1)$ & $22(73.3 \%)$ & 22 \\
\hline Yes & $17(27.9)$ & $8(26.7 \%)$ & 9 \\
\hline \multicolumn{4}{|l|}{ Postoperative metastasis } \\
\hline No & $55(90.2)$ & 28(93.3) & 27 \\
\hline Yes & $6(9.8)$ & $2(6.7)$ & 4 \\
\hline
\end{tabular}

There were 30 males and 31 females with the median age of 51 years (range, 27 to 81 years). The median tumor size was $19 \mathrm{~cm}$ (range, 4.3 to $50 \mathrm{~cm}$ ). According to the FNCLCC grading system, 39 (63.9\%) cases were intermediate-grade and $22(36.1 \%)$ were high-grade. The overall median blood loss was $400 \mathrm{ml}$ (range, 50 to $2700 \mathrm{ml}$ ), with $28(45.9 \%)$ patients losing blood $<400 \mathrm{ml}$, and $33(54.1 \%)$ patients losing blood $\geq$ $400 \mathrm{ml} .51$ (83.6\%) patients received R0/R1 resections while 10 (16.4\%) underwent R2 resections. 30 (49.2\%) patients presented with primary disease, which were comparable to 31 (50.8\%) of recurrent tumors. Besides, all of the 31 patients with recurrent disease were the first recurrence. Tumors were removed intactly in 42 (68.9\%) patients, whereas piecemeal in 19 (31.1\%) patients. Among the 19 patients under went piecemeal resection, 8 were with primary disease. 33 $(54.1 \%)$ patients underwent excisions of at least one adjacent organ, of which the kidney $(n=21,63.6 \%)$ was the most common one. The details of organ resections were shown in Table 2. Patients $(n=42,68.9 \%)$ with unifocal diseases outnumbered those $(n=19,31.1 \%)$ suffered from multifocal disease. Among the 19 patients with multifocality, 6 were with primary disease. Postoperative adjuvant treatment was applied to $17(27.9 \%)$ patients, among whom, $6(9.8 \%)$ received adjuvant radiotherapy, 9 (14.8\%) received chemotherapy, and $2(3.3 \%)$ received both. During the follow-up, postoperative distant metastases were observed in 6 (9.8\%) patients, with 3 to lung, 1 to both lung and oropharynx, 1 to both lung and bone, and 1 to bone.

Table 2. Details of organ resection.

\begin{tabular}{ll}
\hline No. of organ resected & No. (\%) \\
\hline 0 & $28(45.9)$ \\
1 & $13(21.3)$ \\
2 & $9(14.8)$ \\
3 & $8(13.1)$ \\
4 & $3(4.9)$ \\
Organ resected & No. \\
Kidney & $21(63.6)$ \\
Colon & $20(60.6)$ \\
Spleen & $8(24.2)$ \\
Pancreas & $5(15.2)$ \\
Accessory & $4(12.1)$ \\
Psoas major & $4(12.1)$ \\
Small intestine & $1(3.0)$ \\
Adrenal & $2(6.1)$ \\
Stomach & $1(3.0)$ \\
Ureter & $1(3.0)$ \\
\hline
\end{tabular}

\section{Data analysis}

Details of univariate analysis were listed in Table 3. The median duration of follow-up was 49 months (range, 5-134 months). Updating to November of 2017, among the 61 patients, 49 (80.3\%) patients suffered disease relapse. For 51 patients underwent R0/R1 resections, 39 (76.5\%) suffered disease relapse, and $23(45.1 \%)$ died of the disease or its complications. All 10 patients underwent $\mathrm{R} 2$ resection suffered from disease relapse with $8(80 \%)$ died of the disease or its complications. The 5-year OS rate and 5-year PFS rate were $43.5 \%$ and $3.7 \%$, respectively. The time of median OS and median PFS were 58 months (95\% CI 36.1-79.9 months, Fig. 1A) and 19 months (95\% CI 13.1-24.9 months), respectively.

In the univariate analyses, patients with blood loss $<400 \mathrm{ml}$ presented markedly longer median OS compared to patients with blood loss $\geq 400 \mathrm{ml}$ (median 
OS, 72 months, 95\% CI 12.7-131.3 months vs. 48 months, 95\% CI 16.1-79.9 months, $\mathrm{p}=0.014$, Fig. 1B), however, the difference of PFS between the two groups was not statistically significant $(p=0.221)$. Patients with intermediated-grade disease showed favorable OS and PFS compare to those with high-grade disease (median OS: 72 months, 95\% CI 50.2-93.8 months vs. 21 months, 95\% CI 17.3-24.7 months, $\mathrm{p}=0.001$, Fig. 2A; median PFS: 21 months, 95\% CI 16.4-25.6 months vs. 11 months, 95\% CI 5.3-16.7 months, $p=0.045$, Fig. 2B). As for resection extent, the median OS of patients with R0/R1 resection was 60 months (95\% CI 43.3-76.7 months, Fig. 2C), which was significantly longer than that of the patients with R2 resection (median OS, 8 months, 95\% CI 3.4-12.6 months, $\mathrm{p}<0.001$ ). Similar results were also observed in PFS, with the median PFS of $\mathrm{R} 0 / \mathrm{R} 1$ resection group and R2 group being 21 months (95\% CI 13.3-28.7 months, Fig. 2D) and 2 months (95\% CI 0.5-3.5 months, $\mathrm{P}<0.001$ ), respectively. With regard to tumor integrity, intact resection group showed a favorable median OS over piecemeal resection group (median OS, 72 months, 95\% CI 52.9-71.1 months vs. 21 months, 95\% CI 17.0-25.0 months, $p<0.001$, Fig. 3A). Likewise, the median PFS of intact resection group was significantly longer than that of piecemeal resection group (median PFS, 28 months, $95 \%$ CI 18.8-37.2 months vs. 8 months, $95 \%$ CI 2.3-13.7 months, $\mathrm{p}<0.001$, Fig. 3B). Concerning multifocality, unifocal disease group presented an obviously longer PFS compared to multifocal disease group (median PFS, 28 months, 95\% CI 16.6-39.4 months vs. 11 months, $95 \%$ CI 9.3-12.7 months, $\mathrm{p}<$ 0.001 , Fig. 3D), while the difference of OS between the two groups was not statistically significant $(p=0.066$, Fig. 3C). No prominent effects of gender $(p=0.355)$, age (as a categorical variable, $\mathrm{p}=0.731$ ), tumor burden (as a categorical variable, $\mathrm{p}=0.387$ ), admission status $(p=0.777)$, concomitant organ resection $(p=0.504)$ or adjuvant therapy $(\mathrm{P}=0.382)$ were observed in univariate analysis. Because of a wide difference of sample (6 vs. 55), distant metastases were excluded from analysis.
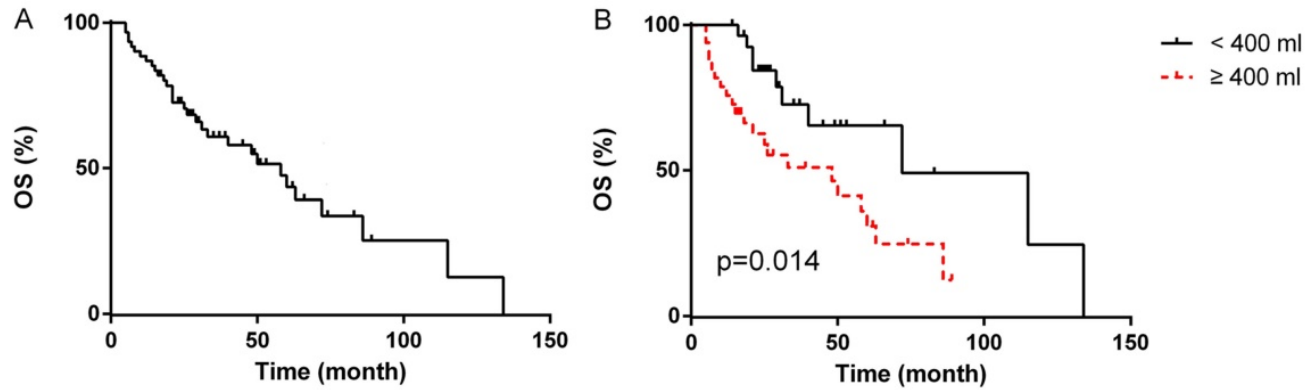

Fig. 1: Overall survival of all patients (A); OS according to blood loss (B)
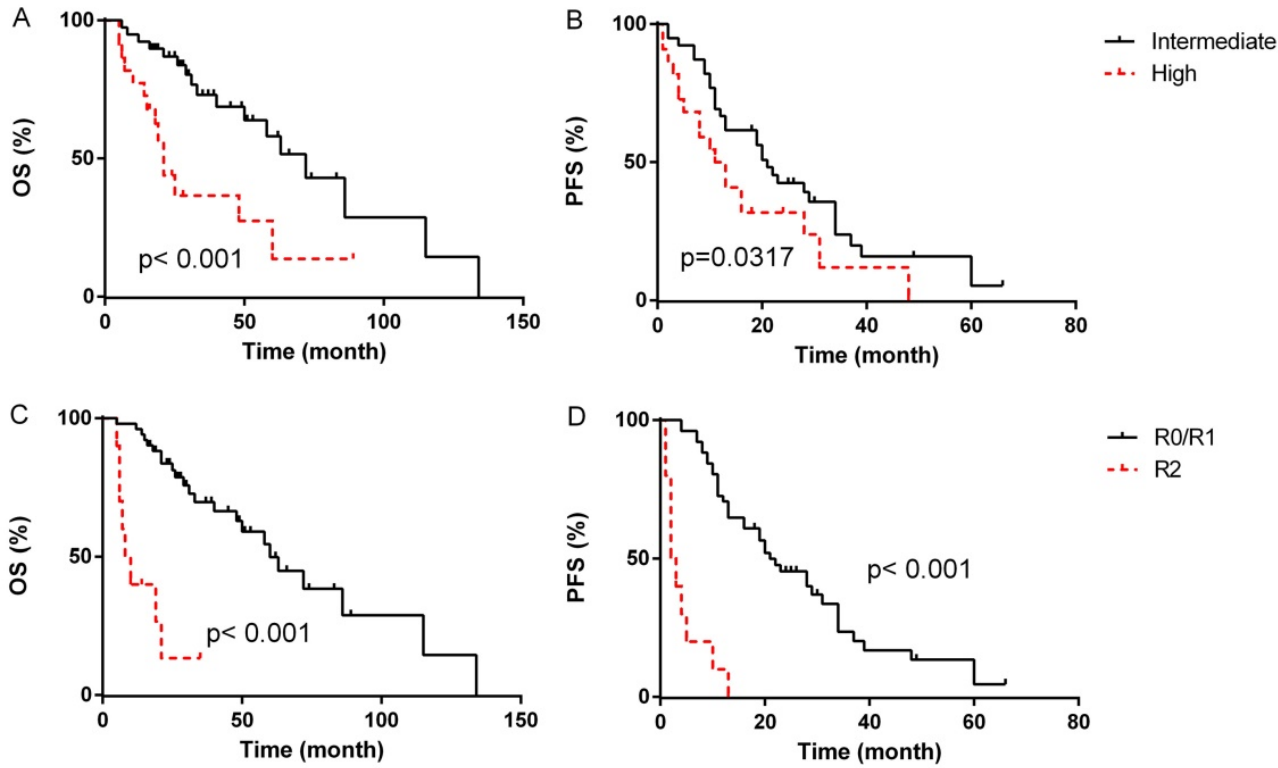

Fig. 2: Survival outcome according to tumor grade (A\&B). Survival outcome according to extent of resection(C\&D). 

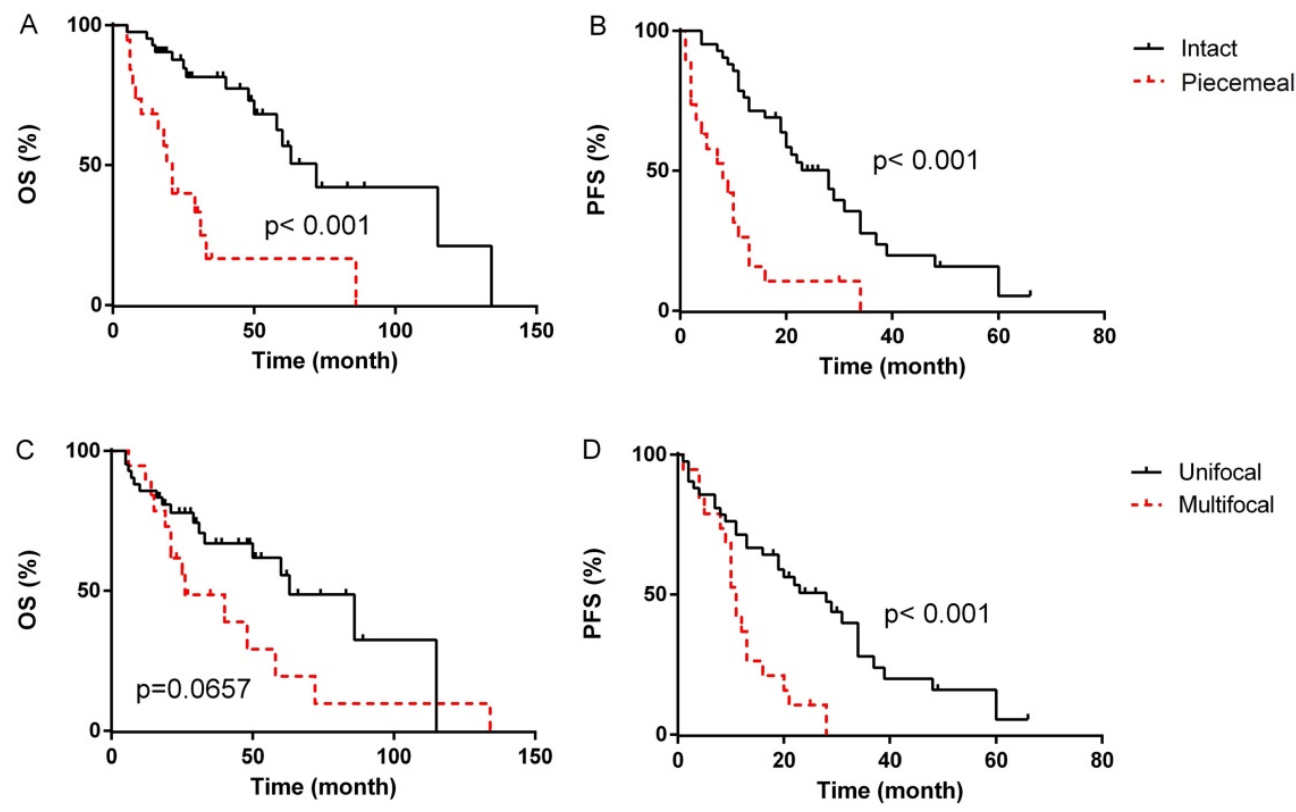

Fig. 3: Survival outcome according to tumor integrity (A\&B) and multifocality (C\&D).

Multivariate analysis using Cox proportional hazards model revealed that tumor grade (HR 2.566, 95\% CI 1.085-6.068, p=0.032), blood loss (HR 3.302, $95 \%$ CI 1.231-8.858, $\mathrm{p}=0.018$ ), extent of resection (HR 4.616, 95\% CI 1.267-16.818, $\mathrm{p}=0.020)$ and tumor integrity (HR 4.043, 95\%CI 1.518-10.768, p=0.005) were significant prognostic factors associated with OS. Meanwhile, tumor grade (HR 2.222, 95\% CI 1.1834.171, $\mathrm{p}=0.013$ ), tumor integrity ( $\mathrm{HR} 3.057,95 \% \mathrm{CI}$ 1.320-7.080, $\mathrm{p}=0.009$ ) and multifocality (HR 2.416, 95\% CI 1.099-5.312, $\mathrm{p}=0.028$ ) were independent prognostic factors for PFS. Details were listed in Table 4.

\section{Discussion}

Liposarcoma is a rare disease prevailingly affecting adults. Accumulating evidence has demonstrated that the clinical outcomes can vary dramatically according to different histologic subtypes and different primary sites $[4,5,12,13]$. Among all the histologic subtypes, DDLS is a high-grade tumor with the systemic metastatic rate of $5 \%$ to $29.7 \%$ [14-16]. There was no previous report focusing specifically on RP DDLS of Asian population. By retrospectively analyzing the data 61 RP DDLS patients from a single center, we sought to describe the clinical features of the disease, and further evaluated the prognostic factors influencing patients' outcome, thus to address this essential knowledge gap.

In this cohort, the 5-year OS, 5-year PFS rate and distant metastatic rate were $43.5 \%, 3.7 \%$, and $9.8 \%$ respectively, which was consistent with those of Keung EZ et al. [9] who analyzed the data of 119 RP DDLS patients.
Because of the deep location and the lack of specific symptoms in early stage, RP LS presented frequently as considerable size by the time of diagnosis. In this cohort, the median size of RP DDLS was $19 \mathrm{~cm}$ (range 4.3-50 cm). Some studies have reported that larger tumor burden was related to worse prognosis [4, 12, 17], while some scholars including Keung EZ et al. showed no difference [5, 9]. In our study, we didn't detect any prognostic significance of tumor burden.

As for FNCLCC tumor grade, several studies reported that high grade was associated with worse disease-specific survival in RP LS [12, 18]. Keung EZ et al. [9] revealed that high grade was correlated with worse OS, while Tirumani SH et al. [16] found an association between tumor grade and distant metastases. In this study, high grade was proven as an independent predicator for both OS (HR 2.566, 95\% CI 1.085-6.068, $\mathrm{p}=0.032$ ) and PFS (HR 2.222, 95\%CI 1.183-4.171, $\mathrm{p}=0.013$ ).

With regard to intraoperative blood loss, the median blood loss was 400ml (range, 50-2700 ml), and blood loss $\geq 400 \mathrm{ml}$ was proven as an independent negative predictor in our study (HR 3.302, 95\% CI 1.231-8.858, $\mathrm{p}=0.018$ ). However, Lehnert $\mathrm{T}$ et al. who retrospectively analyzed the data of 110 retroperitoneal STS patients reported that blood loss > $1500 \mathrm{ml}$ independently predicted worse prognosis [11]. Apparently, the amount of bleeding was markedly smaller in our research. We assumed the main reasons might be (1) different disease entities (DDLS only vs. various kinds of STS) (2) ethnic difference (Chinese vs. European). 
Table 3. Factors influencing OS/PFS in univariate analysis.

\begin{tabular}{|c|c|c|c|c|}
\hline \multirow[b]{2}{*}{ Variable } & \multicolumn{2}{|l|}{ Overall OS } & \multicolumn{2}{|l|}{ Overall PFS } \\
\hline & Median, mo & p Value & Median, mo & $\mathrm{p}$ Value \\
\hline \multicolumn{5}{|l|}{ Gender } \\
\hline Male & 60 & 0.355 & 21 & 0.537 \\
\hline Female & 50 & & 13 & \\
\hline \multicolumn{5}{|l|}{ Age } \\
\hline$<50 y$ & 50 & 0.731 & 12 & 0.527 \\
\hline$\geq 50 \mathrm{y}$ & 60 & & 20 & \\
\hline \multicolumn{5}{|c|}{ Tumor burden } \\
\hline$<19 \mathrm{~cm}$ & 40 & 0.387 & 13 & 0.266 \\
\hline$\geq 19 \mathrm{~cm}$ & 63 & & 20 & \\
\hline \multicolumn{5}{|l|}{ Grade } \\
\hline Intermediate & 72 & 0.001 & 21 & 0.045 \\
\hline High & 21 & & 11 & \\
\hline \multicolumn{5}{|l|}{ Blood loss } \\
\hline$<400 \mathrm{ml}$ & 72 & 0.014 & 19 & 0.221 \\
\hline$\geq 400 \mathrm{ml}$ & 48 & & 13 & \\
\hline \multicolumn{5}{|l|}{ Presentation } \\
\hline Primary & 60 & 0.777 & 20 & 0.289 \\
\hline Recurrent & 50 & & 13 & \\
\hline \multicolumn{5}{|c|}{ Resection extent } \\
\hline R0/R1 & 60 & $<0.001$ & 21 & $<0.001$ \\
\hline $\mathrm{R} 2$ & 8 & & 2 & \\
\hline \multicolumn{5}{|c|}{ Tumor integrity } \\
\hline Intact & 72 & $<0.001$ & 28 & $<0.001$ \\
\hline Piecemeal & 21 & & 8 & \\
\hline \multicolumn{5}{|c|}{ Organ resection } \\
\hline No & 31 & 0.504 & 13 & 0.130 \\
\hline Yes & 60 & & 21 & \\
\hline \multicolumn{5}{|l|}{ Multifocality } \\
\hline No & 63 & 0.066 & 28 & $<0.001$ \\
\hline Yes & 26 & & 11 & \\
\hline \multicolumn{5}{|c|}{ Adjuvant therapy } \\
\hline No & 60 & 0.382 & 16 & 0.786 \\
\hline Yes & 50 & & 20 & \\
\hline Chemo- & 50 & 0.441 & 19 & 0.565 \\
\hline Radio- & 58 & 0.929 & 21 & 0.442 \\
\hline
\end{tabular}

*Median: median OS/PFS; mo: months; y: years; Chemo-: chemotherapy; Radio-: radiotherapy.

Table 4. Factors influencing the OS/PFS in multivariate analysis

\begin{tabular}{|c|c|c|c|c|}
\hline \multirow[b]{2}{*}{ Variable } & \multicolumn{2}{|l|}{ Overall OS } & \multicolumn{2}{|l|}{ Overall PFS } \\
\hline & $\operatorname{HR}(95 \% \mathrm{CI})$ & $\mathrm{p}$ & HR $(95 \%$ CI $)$ & $\mathrm{p}$ \\
\hline $\begin{array}{l}\text { Grade } \\
\text { (intermediate vs high) }\end{array}$ & $\begin{array}{l}2.566 \\
(1.085-6.068)\end{array}$ & 0.032 & $\begin{array}{l}2.222 \\
(1.183-4.171)\end{array}$ & 0.013 \\
\hline $\begin{array}{l}\text { Blood loss } \\
(<400 \mathrm{ml} \mathrm{vs} \geq 400 \mathrm{ml})\end{array}$ & $\begin{array}{l}3.302 \\
(1.231-8.858)\end{array}$ & 0.018 & - & - \\
\hline $\begin{array}{l}\text { Resection extent (R0/R1 } \\
\text { vs R2) }\end{array}$ & $\begin{array}{l}4.616 \\
(1.267-16.818)\end{array}$ & 0.020 & $\begin{array}{l}2.793 \\
(0.884-8.825)\end{array}$ & 0.080 \\
\hline $\begin{array}{l}\text { Tumor integrity (intact } \\
\text { vs piecemeal) }\end{array}$ & $\begin{array}{l}4.043 \\
(1.518-10.768)\end{array}$ & 0.005 & $\begin{array}{l}3.057 \\
(1.320-7.080)\end{array}$ & 0.009 \\
\hline $\begin{array}{l}\text { Multifocality } \\
\text { (No vs Yes) }\end{array}$ & - & - & $\begin{array}{l}2.416 \\
(1.099-5.312)\end{array}$ & 0.028 \\
\hline
\end{tabular}

In terms of admission status, some literatures reported that recurrent tumor was negatively associated with OS or PFS [17, 19]. In the present study, however, we didn't observe any significant effect of the recurrent disease on PFS/OS. We assumed that the following reasons might explain this unexpected result. Firstly, our institution was a tertiary hospital, which meant that the illnesses our patients were generally more serious. The severity of the primary disease might contribute to the indiscrimination of patients' outcome between primary and recurrent group. The tumor burden (median $20 \mathrm{~cm}$, range $7-50 \mathrm{~cm}$ ) and blood loss (median $400 \mathrm{ml}$, range $50-2700 \mathrm{ml}$ ) of the primary disease group were bigger than that of the recurrent disease group (tumor burden: median $18 \mathrm{~cm}$, range $4.3-33 \mathrm{~cm}$; blood loss: median $300 \mathrm{ml}$, range 50-2500 $\mathrm{ml}$ ). Secondly, our patients might be too small to discover the impact of recurrent disease on patients' outcome.

In this study, concomitant organ resections were only performed for directly involved adjacent organs at the time of surgery or to facilitate expected gross tumor resections. The most frequently included organ was kidney $(n=21,63.6 \%)$, which was identical with the study of Keung EZ et al. [9] (kidney, n=78, 65.5\%). In this cohort, no significant effect of organ resection on PFS/OS was observed in this cohort. The result was in line with previous researches, including the studies of Keung EZ et al. and Ikoma N et al. [9, 20].

Owing to the inefficiency of adjuvant therapy, surgical complete resection remains the cornerstone for the treatment of LS. To the best of our knowledge, gross tumor resection is associated with favorable prognosis [5, 9, 12, 15]. In this cohort, patients with R2 resections presented obvious worse OS/PFS compared to patients with R1/R0 resections, and resection extent was proven an independent prognostic factor for OS (HR 4.616, 95\% CI 1.267-16.818, $p=0.020$ ), which was in line with the study of Keung EZ et al. [9].

Due to the deep location, general large size, and proximity to vital structures of RP LS, sometimes it's inevitable to cut through the tumor to achieve gross tumor resection. Some scholars have reported that tumor rupture was associated with worse prognosis [9], an effect that was also observed in our research. In this cohort, both median OS and median PFS of intact resection group were significantly longer than that of piecemeal resection group. Moreover, multivariate analysis demonstrated that tumor integrity was an independent predictor for both OS (HR 4.043, 95\% CI 1.518-10.768, $\mathrm{p}=0.005$ ) and PFS (HR 3.057, 95\%CI 1.320-7.080, $\mathrm{p}=0.009$ ).

So far, the correlation between multifocal disease of RP LS and patients' outcome has not been well expatiated. The incidence multifocality (31\%) in this study was comparable to that of Tseng WW et al.'s research (34\%) [21], which included 105 RP DDLS patients. Tseng WW et al. reported that multifocality was associated with worse OS in primary RP DDLS, yet did not impact OS in recurrent RP DDLS [21]. However, Keung EZ et al. [9] and Anaya DA et al. [22] showed that multifocality was independent negative predicator for local recurrence free survival (DRFS) and OS, respectively. In our study, because of the 
relatively small sample, we didn't study the impact of multifocality on primary and recurrent disease separately. Overall, we found that multifocality was an independent prognostic factor for PFS (HR 2.416, 95\% CI 1.099-5.312, $\mathrm{p}=0.028$ ).

In terms of postoperative adjuvant therapy, the role of chemotherapy and radiotherapy remains a controversial issue in patients with LS. Generally, DDLS relatively chemo-insensitive compared with other histology subtypes of LS [23]. A recent DDLS specific study from MD Anderson Cancer Center reported a response rate of $24 \%$ to combination chemotherapy [24]. Doxorubicin-based regime remains the first-line chemotherapy to metastatic or unresectable disease, although response rates are low. Pre-operative radiation therapy is the preferred method of adjuvant radiation therapy for retroperitoneal LS [25-27]. In this cohort, however, we did not observe significant effect of the chemotherapy or radiotherapy on patients' OS or PFS.

\section{Conclusion}

RP DDLS was a rare disease of deep location, general large size, and frequent proximity to vital structures. R0/R1 resection remained the mainstay of treatment, and was an independent favorable prognostic factor of OS. Blood loss $<400 \mathrm{ml}$ also predicted better prognosis. Surgeons should endeavor to excise the tumor intactly since tumor integrity was an essential predictor for both OS and PFS. Tumor grade and multifocality independently predicted worse PFS. We hope our research may facilitate further prospective studies and clinical decision-making in RP DDLS patients.

\section{Authors' contributions}

Peng Luo, Lingge Yang, Zhiqiang $\mathrm{Wu}$ and Weiluo Cai performed patient recruitment and clinical investigation. Chunmeng Wang, Peng Luo, Wangjun Yan, Yong Chen, Ruming Zhang, Yingqiang Shi conceived of the study, participated in its design and coordination and helped draft the manuscript. All authors read and approved the final manuscript.

\section{Ethics approval and consent to participate}

Written informed consent was obtained from all patients. This study was approved by the ethics committee of Fudan University Shanghai Cancer Center. Each author certifies that all investigations were conducted in conformity with the ethical principles.

\section{Competing Interests}

The authors have declared that no competing interest exists.

\section{References}

1. Crago AM, Singer S. Clinical and molecular approaches to well differentiated and dedifferentiated liposarcoma. Curr Opin Oncol. 2011; 23: 373-378.

2. Evans HL. Liposarcoma: a study of 55 cases with a reassessment of its classification. Am J Surg Pathol. 1979; 3: 507-23.

3. Henricks WH, Chu YC, Goldblum JR, et al. Dedifferentiated liposarcoma: a clinicopathological analysis of 155 cases with a proposal for an expanded definition of dedifferentiation. Am J Surg Pathol. 1997; 21: 271-81.

4. Dalal KM, Kattan MW, Antonescu CR, et al. Subtype specific prognostic nomogram for patients with primary liposarcoma of the retroperitoneum, extremity, or trunk. Ann Surg. 2006; 244: 381-91.

5. Oh YJ, Yi SY, Kim KH, et al. Prognostic Model to Predict Survival Outcome for Curatively Resected Liposarcoma: A Multi-Institutional Experience. J Cancer. 2016; 7: 1174-80.

6. Goldblum JR, Weiss SW, Folpe AL. Enzinger and Weiss's soft tissue tumors (6th Edition). Philadelphia, USA: Saunders; 2013.

7. Henricks WH, Chu YC, Goldblum JR, et al. Dedifferentiated liposarcoma: A clinicopathologic analysis of 155 cases with a proposal for an expanded definition of dedifferentiation. Am J Surg Pathol. 1997; 21: 271-81.

8. Singer S, Antonescu CR, Riedel E, et al. Histologic subtype and margin of resection predict pattern of recurrence and survival for retroperitoneal liposarcoma. Ann. Surg. 2003; 238: 358-70.

9. Keung EZ, Hornick JL, Bertagnolli MM, et al. Predictors of outcomes in patients with primary retroperitoneal dedifferentiated liposarcoma undergoing surgery. J Am Coll Surg. 2014; 218: 206-17.

10. Coindre JM. Grading of soft tissue sarcomas: review and update. Arch Pathol Lab Med 2006; 130: 1448-53.

11. Lehnert $\mathrm{T}$, Cardona $\mathrm{S}$, Hinz $\mathrm{U}$, et al. Primary and locally recurrent retroperitoneal soft-tissue sarcoma: local control and survival. Eur J Surg Oncol. 2009; 35: 986-93.

12. Knebel C, Lenze U, Pohlig F, et al. Prognostic factors and outcome of Liposarcoma patients: a retrospective evaluation over 15 years. BMC Cancer. 2017; 17: 410.

13. Kim HS, Lee J, Yi SY, et al. Liposarcoma: exploration of clinical prognostic factors for risk based stratification of therapy. BMC Cancer. 2009; 9: 205.

14. Huang HY, Brennan MF, Singer S, et al. Distant metastasis in retroperitoneal dedifferentiated liposarcoma is rare and rapidly fatal: a clinicopathological study with emphasis on the low-grade myxofibrosarcoma-like pattern as an early sign of dedifferentiation. Mod Pathol. 2005; 18: 976-84.

15. Ghadimi MP, Al-Zaid T, Madewell J, et al. Diagnosis, management, and outcome of patients with dedifferentiated liposarcoma systemic metastasis. Ann Surg Oncol. 2011; 18: 3762-70.

16. Tirumani $\mathrm{SH}$, Tirumani $\mathrm{H}$, Jagannathan JP, et al. Metastasis in dedifferentiated liposarcoma: Predictors and outcome in 148 patients. Eur J Surg Oncol. 2015; 41: 899-904.

17. Anaya DA, Lahat $G$, Wang $X$, et al. Postoperative nomogram for survival of patients with retroperitoneal sarcoma treated with curative intent. Ann Oncol. 2010; 2: 397-402.

18. Mussi C, Collini P, Miceli R, et al. The prognostic impact of dedifferentiation in retroperitoneal liposarcoma: a series of surgically treated patients at a single institution. Cancer. 2008; 113: 1657-65.

19. Lehnert T, Cardona S, Hinz U, et al. Primary and locally recurrent retroperitoneal soft-tissue sarcoma: local control and survival. Eur J Surg Oncol. 2009 Sep; 35: 986-93.

20. Ikoma N, Roland CL, Torres KE, et al. Concomitant organ resection does not improve outcomes in primary retroperitoneal well-differentiated liposarcoma: A retrospective cohort study at a major sarcoma center. J Surg Oncol. 2018; 117: 1188-1194

21. Tseng WW, Madewell JE, Wei W, et al. Locoregional disease patterns in well-differentiated and dedifferentiated retroperitoneal liposarcoma: implications for the extent of resection? Ann Surg Oncol. 2014; 21: 2136-43.

22. Anaya DA, Lahat G, Liu J, Xing Y. Multifocality in retroperitoneal sarcoma: a prognostic factor critical to surgical decision-making. Ann Surg. 2009; 249: 137-42.

23. Dangoor A, Seddon B, Gerrand C, et al. UK guidelines for the management of soft tissue sarcomas. Clin Sarcoma Res. 2016; 6: 20.

24. Livingston JA, Bugano D, Barbo A, et al. Role of chemotherapy in dedifferentiated liposarcoma of the retroperitoneum: defining the benefit and challenges of the standard. Sci Rep. 2017; 7: 11836.

25. Baldini EH, Wang D, Haas RL, et al. Treatment Guidelines for Preoperative Radiation Therapy for Retroperitoneal Sarcoma: 
Preliminary Consensus of an International Expert Panel. Int J Radiat Oncol Biol Phys. 2015; 92: 602-12.

26. Smith MJ, Ridgway PF, Catton CN, et al. Combined management of retroperitoneal sarcoma with dose intensification radiotherapy and resection: long-term results of a prospective trial. Radiother Oncol. 2014; 110: $165-71$.

27. Molina G, Hull MA, Chen YL, et al. Preoperative radiation therapy combined with radical surgical resection is associated with a lower rate of local recurrence when treating unifocal, primary retroperitoneal liposarcoma. J Surg Oncol. 2016; 114: 814-820. 